\title{
Closed-Loop Tracking Using Multimodal RF/EO Sensors
}

\author{
Sean M. O'Rourke, A. Lee Swindlehurst \\ Center for Pervasive Communications and Computing \\ University of California, Irvine; Irvine, CA 92697 \\ Email: \{orourkes,swindle\}@uci.edu
}

\begin{abstract}
This paper addresses the problem of using multiple, spatially distributed RF/EO sensors for target tracking. Data from the multi-modal sensors is collected at a fusion center, and used to track multiple objects in the sensors' field of view. Continuous approximations are made of the sensors' resolution in range, range rate and azimuth in terms of system parameters such as bandwidth, PRF, EO zoom and pointing angle. These approximations are used to create a continuous objective function in terms of the tracking filter's information matrix, and a search is undertaken to find the system parameters that optimize tracking performance based on the current target environment. Simulations demonstrate the benefit of this technique compared with open-loop tracking methods.
\end{abstract}

\section{INTRODUCTION}

Adaptive tracking system configuration is hardly a novel concept, with a history that spans many decades. Agile beam radars, as well as sensor resource allocation, are discussed extensively in [1] and references therein. Kershaw and Evans [2] focused specifically on waveform adaptivity for RF sensors by performing a direct minimization of the next-state prediction error covariance (and thus, indirectly, the measurment covariance), later extended in [3] to tracking in clutter using the probabalistic data association filter (PDAF). However, both of these works were limited to single-target tracking and a restricted set of transmit waveform patterns, which greatly simplified the required optimization scheme. Further techniques in radar waveform adaptivity are covered in the excellent review article by Sira, et al. [4] and references therein. Adaptation for passive sensor systems has also been considered, e.g. [5].

In addition to system adaptivity, research has investigated the potential benefits and challenges of target tracking with multimodal sensor platforms, i.e. incorporating multiple sensor types into a single platform. Particular emphasis has been placed on the combination of radar and optical sensors canonical examples of active and passive sensor types - for a wide variety of applications, including human gait tracking and analysis [6].

More recently, work has focused on systems fusing adaptivity and multimodal sensing for enhanced multitarget tracking performance. [7] exemplifies this effort, considering a system

This work was supported by the Sensors Directorate of the Air Force Research Laboratory (AFRL/RYRT) under contract number FA8650-08-D1303 to Dynetics, Inc. with multiple joint radar/electro-optical sensor platforms that are independently tuned throughout the tracking period. This is accomplished through a waveform-agile particle filter to track multiple targets in clutter, using heuristic methods to select the system parameter set that minimized the posterior Cramer-Rao lower bound (PCRLB).

Of the aforementioned existing literature, almost none consider the effects of finite sensor field-of-view (FOV) on tracking performance or system adaptability. For example, this effect is particularly pronounced in EO sensors, since a target outside the FOV is essentially unmeasurable. Furthermore, most work has concentrated on controlling precise aspects of (usually active) sensors. Our consideration of multimodal sensor platforms, however, necessitates a system-level perspective on "waveform"-adaptivity in the spirit of Daum [8] nearly twenty years ago, which emphasized the impact of resolution on adaptive parameter schemes for multitarget tracking. Since these realistic constraints exist, this demands that we model and quantify their impact on tracking performance and system parameter adaptability. Naturally, this implies that we also must develop algorithms that select near-optimal parameter sets given these models.

More explicitly, our goals for this work are threefold. First, we formulate realistic representations of sensor measurement error and field-of-view (FOV), in terms of known sensor parameters like resolution, suitable for use in an explicit waveform adaptive optimization scheme. These representations mathematically take the form of a "penalty function" that imposes large measurement variances for target measurements that would be outside a sensor's FOV. Second, we develop error metrics, objective functions, and constraints appropriate for a method to find an optimal set of sensor parameters that incorporate the aforementioned measurement error and FOV representations. Finally, we demonstrate the efficacy of this optimization method, as well as the feasibility of the penalty function representation, in a multiplatform-multitarget environment. The rest of the paper is as follows: Section II describes the target state and measurement models. The concept of penalty functions and their contribution to sensor measurement variance is introduced in Section III, and the overall optimization scheme is presented in Section IV. A numerical simulation of an illustrative target/platform scenario, implementing the aforementioned optimization scheme, is pro- 
vided in Section V. Finally, conclusions about the effectiveness of this method are given in Section VI.

\section{TARGET-STATE DYNAMIC AND PLATFORM MEASUREMENT MODELS}

\section{A. Target dynamic model}

In this work, we consider a common non-maneuvering point-target dynamic model for the targets we wish to track, operating in discrete time with a sampling period $T$. For simplicity, we have only considered two-dimensional movement in the $(x, y)$ plane. At time step $k$, the per-target state vector is comprised of Cartesian position and velocity coordinates:

$$
\mathbf{x}_{k}^{i}=\left[\begin{array}{llll}
x_{k}^{i} & \dot{x}_{k}^{i} & y_{k}^{i} & \dot{y}_{k}^{i}
\end{array}\right]^{T} .
$$

In the above equation, and throughout the rest of this paper, we shall use the superscript (first subscript) $i$ to attribute a parameter to the $i$ th target (sensor platform). If no superscript (first subscript) is given, the variable in question applies to a single target (sensor platform). The state update equation for a single target is given by

$$
\mathbf{x}_{k+1}^{i}=\mathbf{A}_{k}^{i} \mathbf{x}_{k}^{i}+\mathbf{w}_{k}^{i}
$$

where $\mathbf{A}_{k}^{i}$ is the maneuver-dependent system matrix and $\mathbf{w}_{k}^{i}$ is zero-mean Gaussian process noise with covariance matrix $\mathbf{Q}_{k}^{i}$. If we assume that there are $N_{T, k}$ targets at time $k$, then we can stack the individual target states into a single multitarget supervector $\chi_{k}=\left[\mathbf{x}_{k}^{(1) T} \cdots \mathbf{x}_{k}^{\left(N_{T, k}\right) T}\right]^{T}$. This multitarget state vector evolves as

$$
\chi_{k+1}=\mathcal{A}_{k} \chi_{k}+\omega_{k+1}
$$

where $\mathcal{A}_{k}=\operatorname{blockdiag}\left[\mathbf{A}_{k}^{(1)}, \ldots, \mathbf{A}_{k}^{\left(N_{T, k}\right)}\right]$ and $\omega_{k+1}$ is a supervector of process noise components composed similarly to the state supervector with a covariance of $\mathcal{Q}_{k}=$ blockdiag $\left[\mathbf{Q}_{k}^{(1)}, \ldots, \mathbf{Q}_{k}^{\left(N_{T, k}\right)}\right]$. For the purposes of this paper, we assume that $N_{T, k}$ is known a priori and constant for all values of $k$.

\section{B. Sensor platform measurement model}

We consider a system of $N_{p} \mathrm{RF} / \mathrm{EO}$ sensor platforms, spatially distributed throughout the region of interest, that provide measurements to a central tracking system. We assume that each platform makes a full set of measurements of each target, the targets are observed with perfect detection for each sensor, and that the measurements have been associated correctly. While this is certainly an idealization of current practice, our intent is to highlight the benefits/results of the penalty functions on the optimization scheme.

For the RF/EO sensor pairing examined in our simulations, the measurement function corresponds to the RF sensor measuring range and range-rate and the EO sensor measuring azimuth.The RF sensors obtain their respective measurements by matched filtering of target returns in a range-doppler, resulting in range and range-rate estimates for each target. For the $j$ th platform located at coordinates $\left(x_{p, j}, y_{p, j}\right)$, the range $r_{j}^{i}$ to and the range-rate $\dot{r}_{j}^{i}$ of the $i$ th target are

$$
\begin{aligned}
r_{j}^{i} & =\sqrt{\left(x^{i}-x_{p, j}\right)^{2}+\left(y^{i}-y_{p, j}\right)^{2}} \\
\dot{r}_{j}^{i} & =\frac{\dot{x}^{i}\left(x^{i}-x_{p, j}\right)-\dot{y}^{i}\left(y^{i}-y_{p, j}\right)}{r_{j}^{i}} .
\end{aligned}
$$

The EO sensors obtain (in this particular scenario) azimuth measurements from their captured image. Hence, the EO measurement equation is $\eta=\arcsin \left(\frac{\left(x^{i}-x_{p, j}\right)}{r_{j}^{i}}\right)$. For one sensor platform, then, the overall measurement vector for the $i$ th target at time step $k$ is $\mathbf{c}_{j, k}^{i}=\left[\begin{array}{ccc}r_{j}^{i} & \dot{r}_{j}^{i} & \eta_{j}^{i}\end{array}\right]^{T}$.

Each of these measurements is corrupted by zero-mean Gaussian noise with a variance that depends on each sensor's measurement parameters, which can be collected in the parameter vector $\mathbf{p}_{j, k}$. If we assume that the measurements are uncorrelated from sensor to sensor, and further, that multiple measurements from a single sensor are also uncorrelated, then the measurement covariance matrix for each target/platform pair can be written as $\mathbf{R}_{j, k}^{i}=$ $\operatorname{diag}\left(\sigma_{r}^{2}\left(\mathbf{p}_{j, k}\right), \sigma_{\dot{r}}^{2}\left(\mathbf{p}_{j, k}\right), \sigma_{\eta}^{2}\left(\mathbf{p}_{j, k}\right)\right)$. The exact forms of these variances, and their dependence on the platform sensor parameters $\mathbf{p}_{j, k}$, will be discussed below in Section V.

More generally, the $j$ th platform measures the $i$ th target as

$$
\mathbf{y}_{j, k}^{i}=\mathbf{c}_{j}\left(\mathbf{x}_{k}^{i}\right)+\mathbf{v}_{j, k}^{i}
$$

where $\mathbf{c}_{j}\left(\mathbf{x}_{k}^{i}\right)$ is the (possibly non-linear) measurement function and $\mathbf{v}_{j, k}^{i}$ is measurement noise. These measurement noise sequences are assumed to be zero-mean Gaussian with system parameter-dependent covariance $\mathbf{R}_{j}^{i}\left(\mathbf{p}_{j, k}\right)$ that are uncorrelated with both the target process noise and the measurement noise from any other platforms.

We form a stacked vector of the measurements from all platforms for each target as (dropping the time dependence for notational ease) $\mathbf{y}^{(i)}=\left[\mathbf{y}_{1}^{(i) T} \cdots \mathbf{y}_{N_{p}}^{(i) T}\right]^{T}$, and then concatenate these per-target vectors into a supervector $\mathcal{Y}=\left[\mathbf{y}^{(1) T} \cdots \mathbf{y}^{\left(N_{T, k}\right) T}\right]^{T}$. This transforms the state-space measurement equation in 6 into:

$$
\mathcal{Y}_{k}=\mathbf{c}\left(\chi_{k}\right)+\nu_{k}
$$

where $\mathbf{c}\left(\chi_{k}\right)$ is the non-linear measurement function composed of the per-target, per-platform measurement functions $\mathbf{c}_{j}^{i}\left(\mathbf{x}_{k}^{i}\right)$ stacked as above; likewise, $\nu_{k}$ is the similarly generated noise vector with block-diagonal covariance $\mathcal{R}\left(\mathbf{p}_{k}\right)$, where $\mathbf{p}$ is the overall system parameter vector composed of the per-platform parameter vectors $\mathbf{p}_{j}$.

\section{Target-state estimation: Extended Kalman filter}

With both our state and measurement models defined, we now require an appropriate state estimation method. The natural choice, due to the nonlinear measurement equations, is the ubiquitous extended Kalman filter (EKF). While more sophisticated state estimation techniques have been used in the literature, the use of the EKF here is primarily to provide a 
simpler metric formulation for optimization. Using the multitarget state vector $\chi_{k}$ and given the measurement supervector $\mathcal{Y}_{k}$, the EKF runs as follows:

Prediction step:

$$
\begin{aligned}
\hat{\chi}_{k \mid k-1} & =\mathcal{A}_{k} \hat{\chi}_{k-1 \mid k-1} \\
\mathcal{P}(k \mid k-1) & =\mathcal{A}_{k} \mathcal{P}_{k-1 \mid k-1} \mathcal{A}_{k}^{T}+\mathcal{Q}_{k}
\end{aligned}
$$

Update step:

$$
\begin{aligned}
& \hat{\chi}_{k \mid k}=\hat{\chi}_{k \mid k-1}+\mathcal{K}_{k} \mathbf{e}_{k} \\
& \mathcal{P}_{k \mid k}=\left[\mathcal{P}_{k \mid k-1}^{-1}+\mathcal{C}_{k}^{T} \mathcal{R}^{-1}\left(\mathbf{p}_{k}\right) \mathcal{C}_{k}\right]^{-1}
\end{aligned}
$$

where $\mathbf{e}_{k}=\mathcal{Y}_{k}-\mathbf{c}\left(\hat{\chi}_{k \mid k-1}\right)$ is the innovation, $\mathcal{K}_{k}=$ $\mathcal{P}_{k \mid k} \mathcal{C}_{k}^{T} \mathcal{R}^{-1}\left(\mathbf{p}_{k}\right)$ is the Kalman gain, and $\mathcal{C}_{k}$ is the Jacobian of $\mathbf{c}\left(\chi_{k}\right)$ w.r.t. $\chi_{k}$ evaluated at $\hat{\chi}(k \mid k-1)$.

\section{PENAlty FUnCTION FRAMEWORK AND APPROXIMATIONS}

As mentioned previously, in most existing literature (and indeed, in our inital treatment in Section II), the noise variance for each measurement, $\sigma_{\text {meas }}^{2}$, is treated as uniform throughout a validation region and, for the most part, valid for all possible measurement values. In practice, sensors are limited to a finite field-of-view (FOV) for each measurement they provide by characteristics like bandwidth, so the latter assertion does not hold. The extent of this FOV is dictated by the same parameters that define the overall measurement variance, thus constraining the feasible set of signal parameters available to the system at each time step. In [7], Zhang, et al. acknowledge the tradeoff between FOV and measurement accuracy, but do not explicitly model it. In our framework, we explictly model this constraint by adding a penalty function $\phi$ to the measurement variance that assigns large variances to target measurements that would appear outside a particular sensor's FOV. This corresponds to the indicator function-like nature of the constraint: if a target lies within the sensor's FOV, then it is measured with some known variance; targets outside the FOV are not measured at all, which corresponds to an infinite measurement variance. However, since indicator functions are non-differentiable, they are difficult to implement in realistic optimization schemes; hence, we replace the indicator with the penalty function. More formally, we can write this extended concept of the measurement variance as

$$
\sigma_{\text {meas }}^{2}=\sigma_{\text {min }}^{2}(\mathbf{p})+\phi(x, F O V(\mathbf{p}))
$$

where $x$ is the measured quantity and $\mathbf{p}$ is a vector of sensor parameters (e.g., pixel angular subtense) that parameterizes both the minimum measurement variance $\sigma_{\min }^{2}$ and the sensor $F O V$. Simple penalty functions include scaled Heaviside step functions (and continuous approximations thereof, which are discussed below), as well as the "deadzone-linear" function [9]. Descriptions of more complicated penalty functions, like the log-barrier and Huber functions, can be found in [9] and references therein. Note that these formulations need to be shifted and/or scaled appropriately for each sensor's particular operation parameters.
In this paper, we use a naive continuous approximation to the Heaviside step function, as the discontinuous nature of the step function makes less attractive for optimization. The unit step can be approximated using the hyperbolic tangent function [10]:

$$
\begin{aligned}
u\left(x-x_{o}\right) & \approx \frac{1}{2}\left(1+\tanh \left(\nu\left(x-x_{o}\right)\right)\right) \\
& =\left(1+\exp \left(-2 \nu\left(x-x_{o}\right)\right)\right)^{-1} .
\end{aligned}
$$

for large values of $\nu$, a shaping parameter. Examples of the approximation for certain values of $\nu$ are shown in Figure 1, which shows clearly that the larger the shaping parameter, the better the approximation. However, this comes with the caveat that a sharper transition near the FOV boundary is less amenable to numerical optimization schemes. With this approximation, our penalty function now becomes:

$$
\phi(x, F O V(\mathbf{p}))=\frac{\gamma_{x}}{1+\exp (-2 \nu(x-F O V(\mathbf{p})))}
$$

where, again, $\gamma_{x}$ is an appropriately large constant.

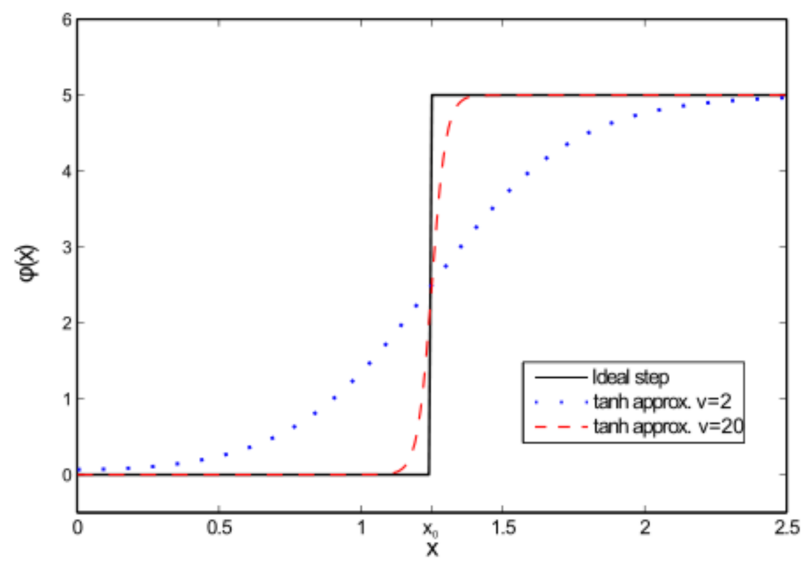

Fig. 1. Hyperbolic tangent approximation to scaled step function

Now that we have an appropriate means of modeling the effect of the finite FOV on measurement variance, we can apply it to the RF/EO system whose measurement equations were described in Section II-B. For the pulse-doppler radar considered, the pulse repetition frequency (PRF) defines the FOV for both the range and range-rate measurements in the form of the maximum unambiguous values $R_{\max }$ and $\dot{R}_{\max }$ :

$$
\begin{array}{cc}
F O V(r)=\left[0, R_{\max }\right] & R_{\max }=\frac{\lambda \mathrm{PRF}}{2} \mathrm{~m} \\
F O V(\dot{r})=\left[0, \dot{R}_{\max }\right] & \dot{R}_{\max }=\frac{c}{2 \mathrm{PRF}} \mathrm{m} / \mathrm{s}
\end{array}
$$

where $\lambda$ is the pulse carrier wavelength and $c$ is the speed of light. Similarly, for a 1-D EO image that is $N_{\text {pix }}$ pixels wide with a detector (pixel) angular subtense (DAS) of $\alpha$ radians, the FOV for the azimuth measurement is:

$$
F O V(\eta)=\left[-\frac{N_{p i x} \alpha}{2}, \frac{N_{p i x} \alpha}{2}\right] \operatorname{rad} .
$$


Since the FOV for the EO sensor is two-sided, the corresponding penalty function will also be "two sided," i.e. the sum of two approximations for each side of the domain.

With the framework given above, we can now incorporate finite field-of-view effects into the tracking scheme by replacing the measurement error covariance matrix $\mathcal{R}_{k}$ in the statespace and EKF formulations above with a modified version, $\tilde{\mathcal{R}}$, whose elements incorporate the penalty function as in 12 . Nominally, we would require this true covariance to carry out any optimization or estimation; however, we cannot directly calculate $\tilde{\mathcal{R}}$ because we do not have access to the target state (and thus the true measurements). Instead, we use an estimate, $\hat{\tilde{\mathcal{R}}}$, that is obtained by evaluating the penalty functions at the predicted next-state measurements $\hat{\mathcal{Y}}_{k+1 \mid k}$. This estimated covariance is then propagated through both the EKF and the optimization scheme discussed below.

\section{PRoposed optimization SCHEME}

The overall goal in any tracking system is to somehow improve tracking performance, which is usually characterized by minimizing the mean-square tracking error. Likewise, our method selects the next-step parameter vector $\mathbf{p}_{k+1}$ that minimizes, in a sense, an equivalent metric: a scalarization of the next-step filtered covariance matrix $\mathcal{P}_{k+1 \mid k+1}$. One commonly proposed scalarization is the trace weighted by an appropriate positive semidefinite matrix $\mathbf{W}$ [2], [11]. The weighting matrix's purpose is assigning importance to particular components of the state vector or targets. An identical method, instead of minimizing the next-step prediction covariance, would be to maximize some scalarization of the predicted information matrix $\mathcal{P}_{k+1 \mid k+1}^{-1}$, or a component thereof [11]. We consider a modification of this cost function below.

It can be shown that, using the properties of the determinant and a Taylor series approximation, maximizing the determinant $\left|\mathcal{P}_{k+1 \mid k+1}^{-1}\right|$ is equivalent to maximizing $\operatorname{tr}\left(\mathcal{P}_{k+1 \mid k} \mathcal{C}_{k+1}^{T} \hat{\tilde{\mathcal{R}}}^{-1}\left(\mathbf{p}_{k+1}\right) \mathcal{C}_{k+1}^{i}\right)$, provided the information increase is not substantially larger than the prediction covariance. This new cost function is also reminiscent of the aforementioned weighted trace of the filtered covariance; here, however, we weight the next-step information increase with the prediction error.

With the cost function in place, we must also consider necessary constraints. While the penalty functions have modeled the effects of finite FOV on measurement variance, they alone are not sufficient to enforce the requirement of keeping all targets in view (to an extent). It is possible for $\mathbf{p}_{k+1}$ to maximize the overall cost function at the expense of providing (almost) no new information about a particular target. We combat this effect by imposing a minimum weighted information constraint; that is, the smallest per-target cost function $\operatorname{tr}\left(\mathcal{P}_{k+1 \mid k}^{(i)} \mathcal{C}_{k+1}^{(i) T} \hat{\tilde{\mathcal{R}}}^{(i)-1}\left(\mathbf{p}_{k+1}\right) \mathcal{C}_{k+1}^{(i)}\right)$ must be above some threshhold $t$. We also assume that the system parameters have maximum \& minimum possible values, contained in the vectors $\mathbf{u}$ and $\mathbf{l}$ respectively. Incorporating the constraints, the final optimization problem is

$$
\max _{\mathbf{p}_{k+1}} \operatorname{tr}\left(\mathcal{P}_{k+1 \mid k} \mathcal{C}_{k+1}^{T} \hat{\tilde{\mathcal{R}}}^{-1}\left(\mathbf{p}_{k+1}\right) \mathcal{C}_{k}\right)
$$

subject to:

$$
\begin{gathered}
\min _{i} \operatorname{tr}\left(\mathcal{P}_{k+1 \mid k}^{(i)} \mathcal{C}_{k+1}^{(i) T} \hat{\tilde{\mathcal{R}}}^{(i)-1}\left(\mathbf{p}_{k+1}\right) \mathcal{C}_{k+1}^{(i)}\right) \geq t \\
\mathbf{l} \preceq \mathbf{p}_{k+1} \preceq \mathbf{u}
\end{gathered}
$$

where $\preceq$ indicates an elementwise inequality.

\section{Simulation Results}

The effectiveness of the adaptive scheme using the stepapproximation penalty described above is demonstrated by the following example simulation results. In constructing our simulation, we have assumed that data association has occured, each sensor detects all targets perfectly, target identifications remain constant (i.e. no confusing one target for another), and the platforms send their measurements to a central processor. We consider a two target, two RF/EO platform scenario for a tracking period of 1000 seconds with a sampling period of 1 second. The sensor platforms - located at $(x, y)$ positions (-1 $\mathrm{km},-1 \mathrm{~km})$ (platform 1) and (1 km, -1km) (platform 2) - are stationary with $\mathrm{X}$-band $(f=10 \mathrm{GHz})$ pulse-doppler radars and one-dimensional 1,000 pixel EO sensors with a DAS between $10^{-5}$ and $10^{-2}$ radians/pixel and a look direction of 0 degrees. At each time step, the RF sensors provide range and range-rate measurements, while the EO sensors provide azimuth measurements, as described in Section II-B. For the $\mathrm{RF}$ sensors, the uncorrected range and range-rate measurement standard deviations are given by $\sigma_{r}=\frac{1}{\sqrt{12}}(c / 2 B)$ and $\sigma_{\dot{r}}=\frac{1}{\sqrt{12}}\left(\lambda \mathrm{PRF} / 2 N_{f f t}\right)$, where $\lambda, c$, and PRF are as above; $N_{f f t}$ is the pulse-doppler integration length; and $B$ is the perpulse bandwidth [12]. For the EO sensors, the uncorrected azimuth measurement standard deviation is $\sigma_{\eta}=\frac{\alpha}{\sqrt{12}}$, where the DAS $\alpha$ is as above, provided the target falls within a single DAS [13]. Each of these uncorrected variances, for both platforms, have assumed that the error is uniformly distributed throughout the appropriate resolution cell/pixel.

Platform 1 was intialized with a pulse repetition frequency (PRF) and RF pulse bandwidth (BW) larger than that of platform 2, but the EO sensor DAS' were identical. The targets initially start at rest at $(-1 \mathrm{~km}, 1 \mathrm{~km})$ (target 1$)$ and $(1 \mathrm{~km}, 1 \mathrm{~km})$ (target 2), and are then perturbed by white noise with multiplicative variance $q=0.01$ in both the $x$ and $y$ directions. Initial position estimates were chosen to be about midway to the actual positions with a large error variance. For comparison, we also simulated the scenario and generated target tracks for a non-adaptive method; that is, where the sensor parameters remained constant.

Figures 2, 3, and 4 summarize the salient results of the simulation. Figure 2 shows the platform positions, the actual target trajectories, as well as the predicted tracks for both the non-adaptive and adaptive EKFs. Initially, the adaptive and non-adaptive tracks are identical, but as the targets progress further from the platforms, the adaptive method is able to 


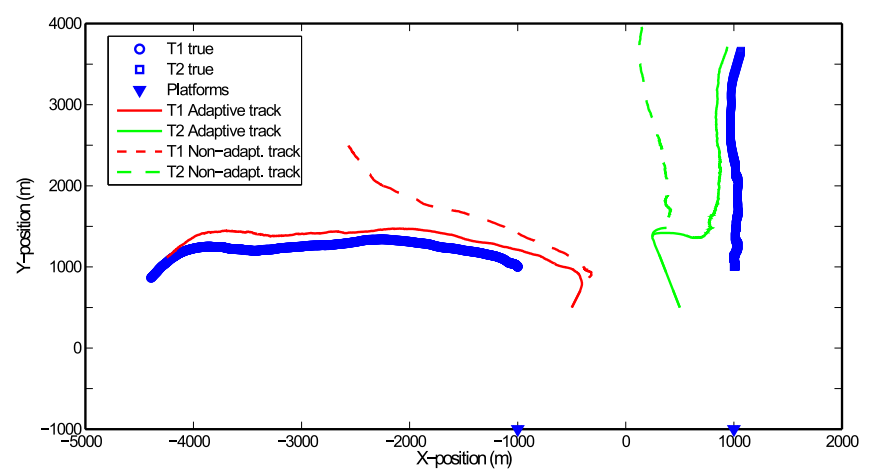

Fig. 2. Actual and estimated (adaptive and non-adaptive) tracking trajectories

maintain tracking while the non-adaptive method is lost. The benefits of the adaptive method are further highlighted in Figure 3, which shows RMS position error for each target and method. The adaptive scheme produces continually decreasing position error for both targets that is at least an order of magnitude less than the non-adaptive results. Figure 4 shows the evolution of the sensor parameters in the adaptive scheme over the tracking period. We can see that the PRF for both platforms follows a ranging technique known for decades [2], where the radar alternates between "low" and "high" PRFs until some quiescent error point is reached. As the targets are relatively close to the platforms, we see little variation in the radar pulse bandwidth. Finally, for the EO sensor, we see that since the motion of Target 2 is essentially within the line of sight of Platform 2, the adaptive scheme assigns this platform the smallest DAS, which corresponds to the smallest FOV, but also the finest resolution. Conversely, as both targets move further apart in azimuth from Platform 1, that platform's EO sensor increases its FOV with a lower DAS. This shows that the scheme favors keeping all targets in view over hyperaccurate measurements of one target at the expense of all others.

\section{CONCLUSION}

We considered a novel constraint on multimodal-multitarget tracking from a system-level perspective. Using penalty function approximations, the effects of finite sensor FOV on target tracking have been modeled. An adaptive system parameter selection criterion, incorporating these penalties, has been constructed. Simulations have demonstrated the benefit of this selection criterion when FOV effects are considered. We anticipate future developments with other penalty functions and objective functions, as well as incorporating FOV effects in tracking situations involving clutter.

\section{REFERENCES}

[1] S. Blackman and R. Popoli, Design and Analysis of Modern Tracking Systems. Norwood, MA: Artech House, 1999.

[2] D. Kershaw and R. Evans, "Optimal waveform selection for tracking systems," IEEE Transactions on Information Theory, vol. 40, no. 5, pp. 1536-1550, 1994.

[3] — "Waveform selective probabilistic data association," IEEE Transactions on Aerospace and Electronic Systems, vol. 33, no. 4, pp. 11801188, Oct. 1997.

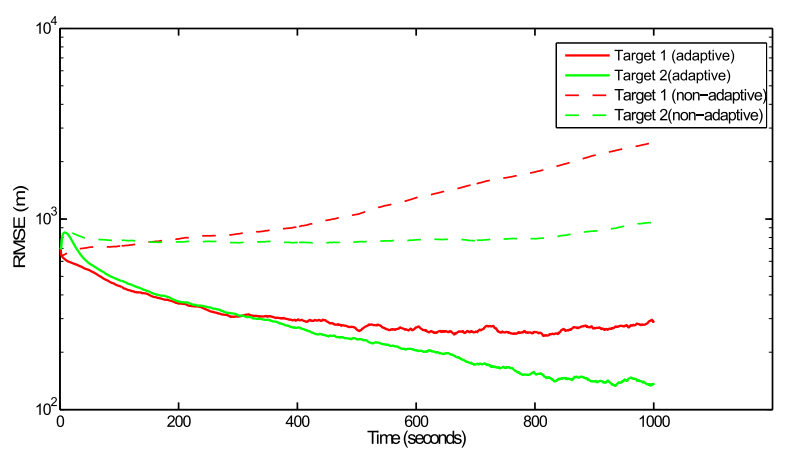

Fig. 3. RMS position error for scenario
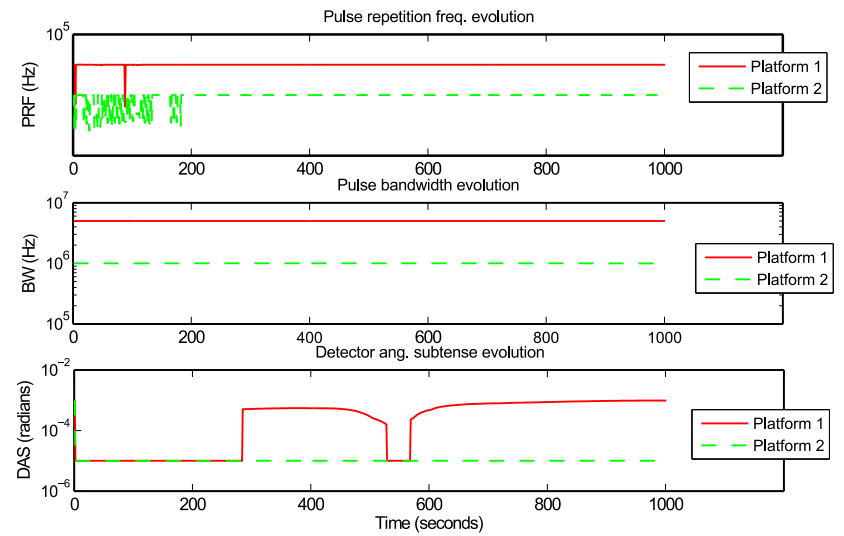

Fig. 4. Evolution of adaptive sensor parameters

[4] S. Sira, Y. Li, A. Papandreou-Suppappola, D. Morrell, D. Cochran, and M. Rangaswamy, "Waveform-agile sensing for tracking," IEEE Signal Processing Magazine, vol. 26, no. 1, pp. 53-64, Jan. 2009.

[5] N. Ukita and T. Matsuyama, "Real-time cooperative multi-target tracking by communicating active vision agents," Computer Vision and Image Understanding, vol. 97, no. 2, pp. 137-179, Feb. 2005. [Online]. Available: http://dx.doi.org/10.1016/j.cviu.2004.07.004

[6] J. M. Blackaby, "Simultaneous RF/EO tracking and characterization of dismounts," 2008.

[7] J. J. Zhang, A. Papandreou-Suppappola, and M. Rangaswamy, "Multitarget tracking using multi-modal sensing withwaveform configuration," in 2010 IEEE International Conference on Acoustics, Speech and Signal Processing. IEEE, Mar. 2010, pp. 3890-3893.

[8] F. Daum, "A System Approach to Multiple Target Tracking," in Multitarget-Multisensor Tracking: Applications and Advances, Volume II, Y. Bar-Shalom, Ed. Norwood, MA: Artech House, 1992, ch. 6, pp. $149-181$.

[9] S. Boyd and L. Vanderberghe, Convex Optimization. Cambridge University Press, 2004.

[10] K. Oldham, J. Myland, and J. Spanier, An Atlas of Functions. New York, NY: Springer New York, 2009. [Online]. Available: http://www.springerlink.com/content/14772727g8383216

[11] D. Casbeer and A. L. Swindlehurst, "A Non-Search Optimal Control Solution for a Team of MUAVS in a Reconnaissance Mission," 2006 IEEE International Conference on Acoustics Speech and Signal Processing Proceedings, no. 1, pp. IV-1169-IV-1172, 2006.

[12] J. P. Stralka and W. G. Fedarko, Pulse Doppler Radar, 3rd ed. McGrawHill, 2008, no. 1990 , ch. 4, pp. 4.1-4.54.

[13] J. Lloyd, "Fundamentals of Electro-Optical Imaging Systems Analysis," in The Infrared \& Electro-Optical Systems Handbook v. 4, M. C. Dudzik, Ed. Ann Arbor, MI \& Bellingham, WA: Infrared Information Analysis Center \& SPIE Optical Engineering Press, 1993, ch. 1, pp. 1-54. 